\title{
Utilizzo di staminali da tessuto adiposo nelle lesioni cutanee croniche: esperienza clinica Use of adipose tissue stem cells in chronic skin lesions: clinical experience
}

\author{
Fabrizio Moffa, Alberico Balbiano da Colcavagno, Elia Ricci \\ U.O.A. Ferite Difficili Casa di Cura e Riposo San Luca, Pecetto Torinese (TO), Italia
}

\section{RIASSUNTO}

La guarigione di una ferita cronica è un processo articolato e complesso, che richiede una visione clinica globale del paziente. Tuttavia, il trattamento locale del letto della ferita riveste un'importanza fondamentale. Sempre più ormai si parla di medicina rigenerativa di tipo induttivo come mezzo per stimolare le cellule senescenti all'interno di una lesione cutanea cronica, in particolare mediante l'utilizzo delle cellule staminali mesenchimali. Il tessuto adiposo autologo rappresenta una fonte di cellule staminali e la tecnologia Lipogems ${ }^{\circledR}$ ne rende possibile la sua applicazione clinica. In questo studio vengono analizzati i risultati ottenuti con tale metodica che, seppur con i limiti di un numero ancora non consistente di pazienti, sono comunque incoraggianti nel proseguire lungo questa strada, considerata da molti come l'ultima frontiera nel wound care.

\section{ABSTRACT}

The healing of a chronic wound is an articulated and complex process, requiring a patient's clinical global vision. However, local treatment of the wound bed is of fundamental importance. More and more now we talk about regenerative medicine as a means to stimulate senescent cells in a chronic skin lesion, particularly through the use of mesenchymal stem cells. Autologous adipose tissue is a source of stem cells and Lipogems ${ }^{\circledR}$ technology makes it possible for its clinical application. In this study we analyze the results obtained with this method that, even with the limits of a not yet substantial number of patients, they are still in encouraging continue along this road, considered by many as the last frontier in wound care.

Corrispondenza: Fabrizio Moffa, U.O.A. Ferite Difficili Casa di Cura e Riposo San Luca, Strada della Vetta 3, 10020 Pecetto Torinese (TO), Italia.

Tel.: 011.8602301.

E-mail: fabrizio.moffa@fastwebnet.it

Parole chiave: Wound care; Stem cells; Healing; Adipose tissue.

Contributi: FM ha reclutato pazienti, disegnato lo studio, eseguito alcuni interventi, scritto il lavoro; $\mathrm{ABC}$ ha reclutato i pazienti, eseguito alcuni interventi, raccolto i risultati; ER ha reclutato pazienti, eseguito alcuni interventi, raccolto i risultati. Tutti gli autori hanno letto e approvato il manoscritto finale.

Conflitto d'interesse: gli autori dichiarano l'assenza di conflitti d'interesse.

Fondi: nessuno.

Ricevuto per la pubblicazione: 24 marzo 2018.

Accettato per la pubblicazione: 9 aprile 2018 .

This work is licensed under a Creative Commons Attribution NonCommercial 4.0 License (CC BY-NC 4.0).

(C) Copyright F. Moffa et al., 2018

Licensee PAGEPress, Italy

Italian Journal of Wound Care 2018; 2(2):37-39

doi:10.4081/ijwc.2018.24

\section{INTRODUZIONE}

Il processo di guarigione atteso nella ferita acuta si interrompe nelle ulcere cutanee arrivando così ad una cronicizzazione del letto di ferita. Pur in un quadro di approccio olistico e globale, si deve tuttavia intervenire localmente determinando una modifica dell'ambiente tale da favorire la riepitelizzazione. ${ }^{1}$ Con l'aumento della patologia una serie di centri specialistici e dedicati stanno trovando una progressiva diffusione sul panorama italiano. Questi centri spesso tendono a sperimentare nuovi presidi, proprio in quanto a essi affluisce una popolazione di pazienti sempre più complessa e difficile. Abbiamo analizzato la nostra esperienza nell'uso delle cellule staminali mesenchimali estratte dal tessuto adiposo attraverso un sistema dedicato.

Il sistema Lipogems ${ }^{\circledR}$ è un sistema di lipoaspirazione, processazione e innesto di tessuto adiposo. ${ }^{2}$ L'intera procedura avviene in un unico tempo chirurgico in anestesia generale o epidurale. Si aspirano circa $60 \mathrm{cc}$ di tessuto adiposo dal grasso comunemente presente a livello dell'addome o della coscia. Il tessuto così prelevato viene introdotto in un sistema chiuso e asettico. Attraverso una minima processazione di tipo meccanico enzyme free si ottiene un tessuto adiposo microfratturato non espanso de- 
stinato ad un uso autologo. ${ }^{3}$ Il prodotto Lipogems ${ }^{\circledR}$ così ottenuto, che ha lo scopo di favorire il naturale processo rigenerativo dei tessuti, ${ }^{4}$ viene iniettato nel fondo e nei bordi della lesione ulcerosa dove svolgendo così un'azione di lipofilling (Figura 1).

\section{MATERIALI E METODI}

Abbiamo raccolto la casistica presso il nostro Centro dei casi trattati con questa tecnica nel periodo che va da

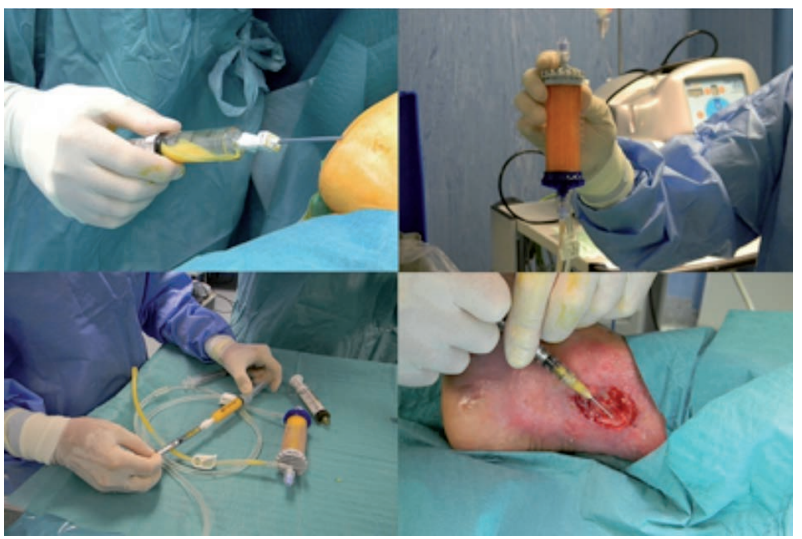

Figura 1. Procedura di lipofilling.

Tabella 1. Anagrafica dei pazienti coinvolti nello studio.

\begin{tabular}{lc}
\hline Pazienti & 17 \\
\hline Maschi & $8(47,1 \%)$ \\
\hline Femmine & $9(52,9 \%)$ \\
\hline Età media pazienti (anni) & $65,01(38 \div 84)$ \\
\hline Età media lesioni (mesi) & $116,3(1 \div 588)$
\end{tabular}

Tabella 2. Eziologia.

\begin{tabular}{ll}
\hline Venose + arteriose & 6 \\
\hline Infiammatorie & 5 \\
\hline Decubito & 4 \\
\hline Altro & 2 \\
\hline
\end{tabular}

Tabella 3. Risultati e stato delle lesioni.

\begin{tabular}{lll}
\hline Migliorata & 6 & $35,2 \%$ \\
\hline Peggiorata & 2 & $11,8 \%$ \\
\hline Invariata & 2 & $11,8 \%$ \\
\hline Risolta & 7 & $41,2 \%$
\end{tabular}

gennaio a giugno 2017. In totale sono stati trattati 17 pazienti, di cui 9 di sesso femminile e 8 di sesso maschile. L'età media dei pazienti è risultata essere di 65,01 anni mentre il tempo medio di insorgenza della lesione era di 116,3 mesi (Tabella 1). Riguardo l'eziologia, il 35\% è rappresentato da ulcere vascolari, il 29\% da ulcere infiammatorie e il 23\% da lesioni da decubito (Tabella 2).

La metodica è stata impiegata in particolare su ulcere profonde, allo scopo di ottenere un riempimento della perdita di sostanza. Inoltre le lesioni presentavano un letto di ferita deterso e granuleggiante e assenza di infezione.

\section{RISULTATI}

Tutti i pazienti sono stati controllati a 4 settimane di distanza. In 7 pazienti ( $41,2 \%$ dei casi) si è osservata la completa risoluzione della lesione. In 6 casi $(35,2 \%)$ è stato evidente un miglioramento della lesione (Figure 2 e 3 ); in

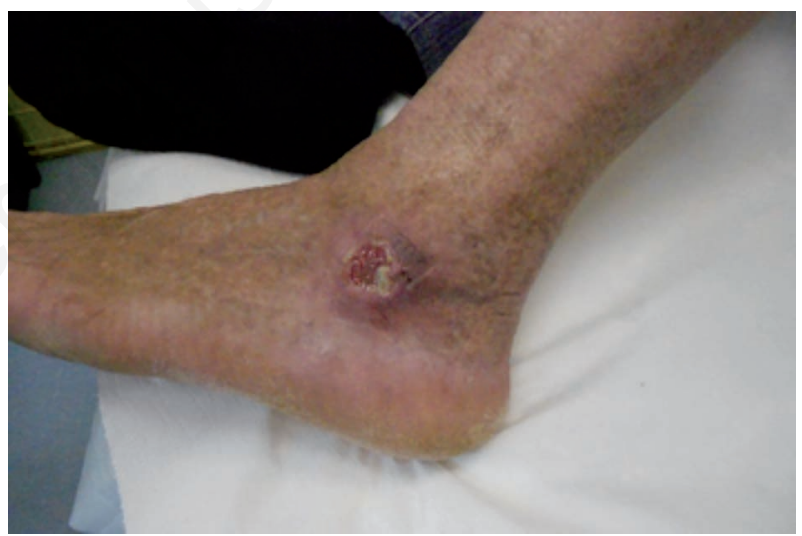

Figura 2. Controllo a 7 giorni dalla somministrazione di Lipogems ${ }^{\circledR}$.

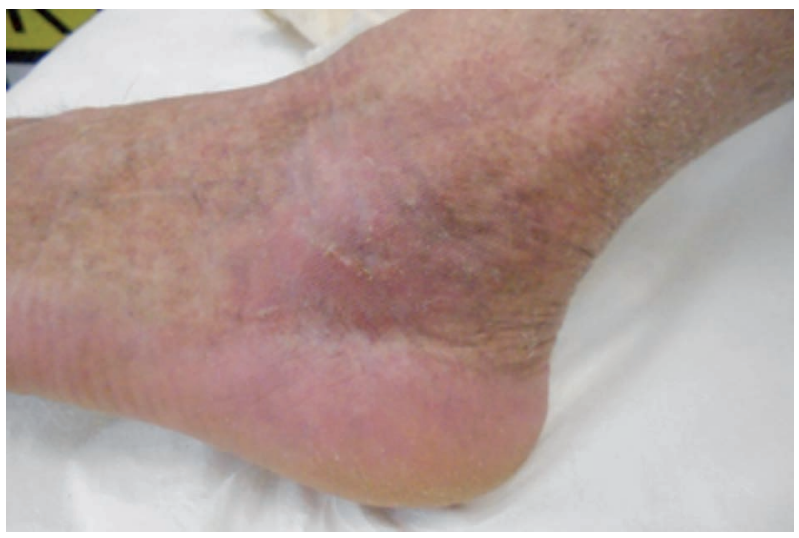

Figura 3. Controllo a 28 giorni dalla somministrazione di Lipogems ${ }^{\circledR}$. 
2 casi non vi è stato alcun risultato; infine in 2 casi abbiamo riscontrato un peggioramento con incremento dell'area della lesione e della situazione locale: in un caso per un'infezione postoperatoria, nel secondo non è stata rilevata una causa specifica (Tabella 3). Il risultato migliore si è ottenuto nel gruppo delle lesioni da decubito, con 3 lesioni su un totale di 4 risolte a 4 settimane mentre la quarta si è risolta a 9 settimane.

\section{DISCUSSIONE}

Abbiamo iniziato a utilizzare tale metodica da gennaio 2017. La tecnica prevede il prelievo del grasso dalla regione addominale (liposuzione): il fatto di dover eseguire tale fase in narcosi o in anestesia epidurale limita la possibilità di reclutamento di pazienti. Inoltre, nel paziente candidato a tale metodica il tessuto adiposo deve essere ben rappresentato, e di conseguenza non può essere utilizzata su soggetti defedati o particolarmente magri (come spesso si riscontra nei pazienti anziani). Sebbene per i motivi sopra esposti lo studio sia stato limitato a pochi soggetti, tuttavia i risultati sono stati molto incoraggianti. Altri studi sono necessari per confermare tali evidenze.

\section{CONCLUSIONI}

L'utilizzo di cellule staminali mesenchimali da grasso risulta essere una valida soluzione nel trattamento di lesioni profonde, per la capacità di riempimento del letto di ferita ed al tempo stesso di rigenerazione tissutale. I limiti risiedono nell'invasività della tecnica, che richiede un'anestesia maggiore, e nella necessità che il paziente disponga di una adeguata quantità di tessuto adiposo. Indubbiamente le tecniche di rigenerazione cellulare che sfruttano l'utilizzo delle cellule staminali mesenchimali stanno emergendo sempre più come una nuova potenziale applicazione clinica nel trattamento delle lesioni cutanee croniche.

\section{BIBLIOGRAFIA}

1. Keast DH, Orsted H. The basic principles of wound care. Ostomy Wound Manage 1998;44:24-31.

2. Tremolada C. Mesenchymal stem cells and regenerative medicine: how Lipogems technology make them easy, safe and more effective to use. MOJ Biol Med 2017;2:00047.

3. Carelli S, Messaggio F, Canazza A, et al. Characteristics and properties of mesenchymal stem cells derived from microfragmented adipose tissue. Cell Transplant 2015;24:1233-52.

4. Kokai LE, Marra K, Rubin JP. Adipose stem cells: biology and clinical applications for tissue repair and regeneration. Transl Res 2014;163:399-408. 